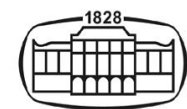

AKADÉMIAI KIADÓ

\title{
Correlation of biofilm formation and antibiotic resistance among clinical and soil isolates of Acinetobacter baumannii in Iraq
}

\section{Acta Microbiologica et Immunologica Hungarica}

67 (2020) 3, 161-170

DOI: 10.1556/030.66.2019.026

(c) 2019 Akadémiai Kiadó, Budapest

\author{
PAKHSHAN A. HASSAN ${ }^{1 *}$ and ADEL K. KHIDER ${ }^{2}$ \\ ${ }^{1}$ Department of Biology, College of Science, Salahaddin University, Erbil, Iraq \\ ${ }^{2}$ Department of Biology, College of Education, Salahaddin University, Erbil, Iraq
}

Received: March 28, 2019 • Accepted: July 17, 2019
ORIGINAL ARTICLE

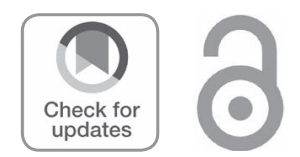

* Corresponding author: Pakhshan Abdulla Hassan Department of Biology, College of Science, Salahaddin University, Erbil 44001, Iraq

Phone: +964 7504612335

E-mail: pakhshanhassana@gmail.com

\begin{abstract}
Acinetobacter baumannii is an opportunistic pathogen that is reported as a major cause of nosocomial infections. The aim of this study was to investigate the biofilm formation by A. baumannii clinical and soil isolates, to display their susceptibility to 11 antibiotics and to study a possible relationship between formation of biofilm and multidrug resistance. During 8 months period, from June 2016 to January 2017, a total of 52 clinical and 22 soil isolates of A. baumannii were collected and identified through conventional phenotypic, chromo agar, biochemical tests, API 20E system, and confirmed genotypically by PCR for $b l a_{\mathrm{OXA}-51-\text { like }}$ gene. Antibiotic susceptibility of isolates was determined by standard disk diffusion method according to Clinical and Laboratory Standard Institute. The biofilm formation was studied using Congo red agar, test tube, and microtiter plate methods. The clinical isolates were $100 \%$ resistance to ciprofloxacin, ceftazidime, piperacillin, $96.15 \%$ to gentamicin, $96.15 \%$ to imipenem, $92.31 \%$ to meropenem, and $78.85 \%$ to amikacin. The soil A. baumannii isolates were $100 \%$ sensitive to imipenem, meropenem, and gentamicin, and $90.1 \%$ to ciprofloxacin. All A. baumannii isolates (clinical and soil) were susceptible to polymyxin B. The percentage of biofilm formation in Congo red agar, test tube, and microtiter plate assays was $10.81 \%, 63.51 \%$, and $86.48 \%$, respectively. More robust biofilm former population was mainly among non-MDR isolates. Isolates with a higher level of resistance tended to form weaker biofilms. The soil isolates exhibited less resistance to antibiotics than clinical isolates. However, the soil isolates produce stronger biofilms than clinical isolates.
\end{abstract}

\section{KEYWORDS}

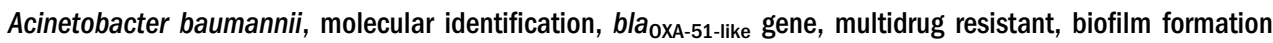

\section{INTRODUCTION}

Acinetobacter baumannii is an opportunistic pathogen that can pose a significant threat due to its increasing antibiotic resistance among nosocomial isolates, mainly from intensive care units [1]. It is related to mainly immunocompromised patients, and a wide range of clinical complications can occur [2]. The plastic genome of A. baumannii, which mutates rapidly when faced with severe conditions, and fundamental mechanisms of virulence beyond canonical drug resistance enable A. baumannii to flourish successfully in the healthcare environment [3]. Biofilm formation is another potent way for bacterial survival in the presence of antibiotics [4], particularly for A. baumannii, which is one of the most common bacterial causes of biofilm-associated contamination of medical devices [5]. Biofilm is known as a structured consortium of microbial cells embedded in a self-produced polymeric matrix or exopolymeric substances, consisting of protein, extracellular DNA, and polysaccharide. Bacterial biofilms are resistant to antibiotics, and they can develop resistance to chemicals, phagocytosis, and other components of the innate and acquired immune system of the body $[6,7]$. Hence, it is important to create connections between biofilm formation and drug resistance in isolates of $A$. baumannii. Therefore, in this study, we focused on determination of biofilm formation in clinical and soil isolates of $A$. baumannii, clarification of their sensitivity 
to different antibiotics, and presenting any possible link between the ability to form biofilm and multidrug resistance (MDR).

\section{MATERIALS AND METHODS}

\section{Collection, isolation, and identification of $A$. baumannii isolates}

A total of 320 clinical samples were taken from patients of three hospitals in Erbil city, Iraq, during June 2016 to January 2017. All clinical specimens were obtained from various sites, which included tracheal aspirate, sputum, burned wound, urine, cerebrospinal fluid, and blood. Specimens were inoculated in brain-heart infusion broth (BHIB; Himedia, India) medium and then transferred to lab for culture. Samples of soil were obtained from different locations in Erbil. Tenfold serial dilutions of soil suspension were made in BHIB, and inocula $(0.1 \mathrm{ml})$ from each dilution were spread with sterile glass beds onto brain-heart infusion agar (BHIA; Himedia) plates. All isolates (clinical and soil) were initially inoculated on MacConkey agar, blood agar, Herellea agar, and on the CHROMagar Acinetobacter Media (CHROMagar, France). Incubation was performed for $24 \mathrm{~h}$ at $37^{\circ} \mathrm{C}$. Standard laboratory methods (Gram-stain and colonial morphology) and conventional biochemical tests (catalase, oxidase, urea test, citrate test, indole test, and the reaction in triple sugar iron medium and growth in $44{ }^{\circ} \mathrm{C}$ ) were used to identify $A$. baumannii. Bacterial species identification of the isolates was confirmed by API 20E system (bioMerieux, France) and by PCR detection of intrinsic $b l a_{\text {OXA-51-like }}$ carbapenemase gene from these isolates.

\section{Molecular identification of isolates by PCR}

All isolates were subjected to conventional PCR to detect

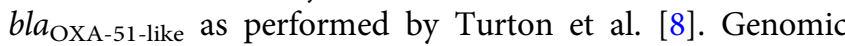
DNA was extracted via a commercial genomic DNA purification kit (Geneaid, Taiwan), according to the manufacture's protocol. Extracted DNA was quantified using the nanodrop spectrophotometer (Thermo Scientific, UK) at OD260 and the purity was determined by OD260/OD280 ratio. The primers were F-TAATGCTTTGATCGGCCTTG and TGGATTGCACTTCATCTTGG (353 bp) [9].

The PCR was carried out in $20 \mu \mathrm{l}$ of reaction volumes (Accua Power PreMix, Bioneer, Korea) with $3 \mu \mathrm{l}$ of extracted DNA, $1 \mu \mathrm{l}$ of forward primer $(10 \mathrm{pmol} / \mu \mathrm{l}), 1 \mu \mathrm{l}$ reverse primer $(10 \mathrm{pmol} / \mu \mathrm{l})$, and $15 \mu \mathrm{l}$ distilled water. The content of PreMix (blue pellet): Top DNA polymerase (1U), dNTP (dATP, dCTP, dGTP, and dTTP): each $250 \mu \mathrm{M}$, reaction buffer, with $1.5 \mathrm{mM}$ of $\mathrm{MgCl}_{2}(1 \times)$. Conditions for the PCR were the following: initial denaturation at $94^{\circ} \mathrm{C}$ for $5 \mathrm{~min}$ and then 30 cycles at $94{ }^{\circ} \mathrm{C}$ for $25 \mathrm{~s}, 52^{\circ} \mathrm{C}$ for $40 \mathrm{~s}$, and at $72{ }^{\circ} \mathrm{C}$ for $50 \mathrm{~s}$, followed by a final elongation at $72{ }^{\circ} \mathrm{C}$ for $6 \mathrm{~min}$. Negative control of sterile distillated water was included in every PCR. A. baumannii ATCC 19606 (purchased from
Media Diagnostic Center) was used as the reference strain. A 100-bp DNA ladder (Norgen, Canada) was used to evaluate the size of PCR products, which were resolved on $1.5 \%$ agarose gels in Tris/borate/EDTA buffer, stained with ethidium bromide, visualized under UV transilluminator (ISOGEN, Life Science, Netherlands) and selected gels were photographed.

\section{Genotypic and phenotypic determination of metallo-beta-lactamases (MBLs)}

Multiplex PCR techniques were used for amplification of the $b l a_{\mathrm{VIM}}, b l a_{\mathrm{IMP}}$, and $b l a_{\mathrm{SIM}}$ genes. Modified Hodge test and imipenem-EDTA-combined disk test (IMP-EDTA-CDT) were performed phenotypically for identification of MBLs in clinical and soil isolates.

\section{Antimicrobial susceptibility test}

Antimicrobial susceptibility test was performed for 11 different therapeutically pertinent antibiotics by the Kirby-Bauer disk diffusion method as stated by Clinical Laboratory Standards Institute guidelines [10]. Antibiotics tested included amikacin (AK; $10 \mu \mathrm{g}$ ), meropenem (MEM; $10 \mu \mathrm{g}$ ), IMP $(10 \mu \mathrm{g})$, polymyxin B (100 U), ceftazidime (CAZ; $30 \mu \mathrm{g})$, amoxicillin/clavulanic acid (AMC; 20/10 $\mu \mathrm{g})$, trimethoprim (TMP; $10 \mu \mathrm{g}$ ), ciprofloxacin (CIP; $10 \mu \mathrm{g})$, piperacillin (PRL; $10 \mu \mathrm{g})$, gentamicin (GEN; $10 \mu \mathrm{g}$ ), and aztreonam (ATM; $10 \mu \mathrm{g})$. The antibiogram procedure performed on MuellerHinton agar swabbed with $1.5 \times 10^{8} \mathrm{CFU}$ of bacterial suspension, equivalent to $0.5 \mathrm{McF}$ arland standard suspension, and incubated for $24 \mathrm{~h}$ at $37^{\circ} \mathrm{C}$. Guidelines from the CLSI were used to interpret the diameters of inhibition zones. Strains resistance to at least three classes of antimicrobial agents (all penicillins and cephalosporins, including inhibitor combinations), aminoglycosides and fluoroquinolones, were defined as MDR Acinetobacter spp., whereas those resistant to the three classes of antimicrobial drugs mentioned above as MDR and resistant to carbapenems were defined as extensive drugresistant (XDR) [11]. MDR A. baumannii (MDRAB) was also detected by CHROMagar by adding MDR Selective supplement CR102 (CHROMagar, Paris, France). A. baumannii ATCC 19606 was used as a non-MDR strain for comparison.

\section{Biofilm formation: Tube method (TM)}

All isolates have been screened qualitatively for the biofilm formation by TM as described by Christensen et al. [12]. Ten milliliters of trypticase soy broth with $1 \%(\mathrm{w} / \mathrm{v})$ glucose in tubes were inoculated with a loop full of test organisms from overnight culture plates. The inoculated tubes were incubated for $24 \mathrm{~h}$ at $37^{\circ} \mathrm{C}$. The tubes were then decanted and washed with phosphate buffer saline (PBS) and stained with crystal violet $(0.1 \%)$ for $10 \mathrm{~min}$. Excess stain was removed and washed with water. The tubes were then mainitained in an inverted position for drying. Biofilm production was detected by the presence of visible film lined the wall and the bottom of the tube. The biofilms were visualized and the amount of biofilm 
formed was recorded according to the result of control strains and graded as $0,1,2$, and 3 representing absent, weak, moderate, and robust biofilm formations, respectively. Experiments were performed two times.

\section{Tissue culture plate (TCP) method}

This quantitative assay described by Christensen et al. [13] is considered as the gold standard method for detection of biofilm formation [14]. An overnight culture of the isolate from fresh agar plate was inoculated into $10 \mathrm{ml}$ trypticase soy broth with $1 \%$ glucose and incubated for $24 \mathrm{~h}$ at $37^{\circ} \mathrm{C}$. The cultures were diluted 1 in 100 with fresh medium and loaded into 96-well-flat bottom - polystyrene TCPs (Coster, USA). Individual wells were filled with $200 \mu \mathrm{l}$ of the diluted cultures ( 3 wells for each isolate), and only sterile broth served as negative control. A. baumannii ATCC 19606 (used as positive control) was also incubated, diluted, and added to the microtiter plate. Plates were covered and incubated for $24 \mathrm{~h}$ at $37^{\circ} \mathrm{C}$ in aerobic condition without shaking. After incubation, the wells were gently decanted by tapping the plate and washed three times with $200 \mu \mathrm{l}$ of PBS ( $\mathrm{pH}$ 7.2) to remove free-floating bacteria. Biofilm formed by adherent bacteria (sessile) in the plate was fixed for $15 \mathrm{~min}$ by $99 \%$ methanol and then stained for $20 \mathrm{~min}$ with crystal violet $(0.1 \% \mathrm{w} / \mathrm{v})$. Excess stain was rinsed off using deionized water and plates were kept for drying. Finally, 95\% ethanol was added to the wells to extract the stain and adherence of the stained cells to the wells. The optical density (OD) of each well was measured at $490 \mathrm{~nm}$ using an automated ELISA plate reader (BioTEK, UK). The triplicate experiment was conducted and the results were averaged. The mean OD at $490 \mathrm{~nm}\left(\mathrm{OD}_{490}\right)$ of sterile trypticase soy broth was used as OD cut-off value $(\mathrm{ODc})$. The $\mathrm{OD}$ results of all tested strains were divided into the following four groups: (1) $\mathrm{OD} \leq \mathrm{ODc}=$ non-biofilm Producer; (2) $\mathrm{ODc}<\mathrm{OD} \leq 2 \times \mathrm{ODc}=$ weak biofilm producer; (3) $2 \times \mathrm{ODc}<\mathrm{OD} \leq 4 \times \mathrm{ODc}=$ moderate biofilm producer; and (4) $4 \times \mathrm{ODc}<\mathrm{OD}=$ strong biofilm producer [15].

\section{Congo red agar method (CRA)}

It is a qualitative method used for screening biofilm formation. As described by Freeman and Keane [16], this method requires CRA media. This medium composed of $37 \mathrm{~g} / \mathrm{L} \mathrm{BHIB,} 50 \mathrm{~g} / \mathrm{L}$ sucrose, $10 \mathrm{~g} / \mathrm{L}$ agar, and Congo red stain $0.8 \mathrm{~g} / \mathrm{L}$. Congo red stain solution was prepared as a concentrated aqueous solution and autoclaved for $15 \mathrm{~min}$ at $121^{\circ} \mathrm{C}$ separately. Then, it was added to autoclaved BHIA (BHIB, agar, and sucrose) at $55^{\circ} \mathrm{C}$. Plates were inoculated with the test bacteria and incubated aerobically at $37^{\circ} \mathrm{C}$ for $24-48 \mathrm{~h}$. A positive result was indicated by black, rough colonies with a dry crystalline consistency; pink, smooth colonies with occasional darkening at the center were recorded as non-biofilm producers.

\section{Statistical analyses}

OD values were expressed either as median values [interquartile range (IQR)] or as means \pm standard deviations (SDs) based on the distribution of variance. All statistical analyses were carried out using the GraphPad prism 6.01. The difference of biofilm biomass between two antibiotics was compared by using Mann-Whitney $U$ test. The values of $p<0.05$ were considered statistically significant.

\section{RESULTS}

\section{Bacterial identification}

Of the 350 clinical specimens and 50 soil samples, 52 and 22 isolates were identified as A. baumannii, respectively. All isolates were first recognized phenotypically on blood agar, MacConkey agar, CHROMagar Acinetobacter, and Herellea agar (Figure 1A-D), then confirmed by API 20E (Figure 1E).

Analysis for the presence of bla OXA-51-like gene presented that all identified isolates were positive and $A$. baumannii was confirmed (Figure 2).

The clinical isolates of $A$. baumannii were recovered from tracheal aspirate $(32.69 \%)$, sputum $(28.85 \%)$, burned wound $(21.15 \%)$, and urine $(9.61 \%)$, and the lowest numbers of isolates $2(3.85 \%)$ were from both blood and cerebrospinal fluid (Figure 3).

\section{Antibiotic susceptibility}

Distribution of antimicrobial resistance properties within the A. baumannii isolates from clinical and soil samples is shown in Table I and Figure 4. As observed, all clinical and soil isolates $(100 \%)$ were resistance to AMC acid. All clinical isolates were resistant to CIP, CAZ, and PRL, but the rate of resistance in soil isolates for these antibiotics was $9.09 \%$, $63.64 \%$, and $90.01 \%$, respectively. IMP and MEM resistance were 96.15 vs. $0 \%$ and 92.31 vs. $0 \%$ in clinical and soil isolates, respectively. All isolates (clinical and soil) were sensitive to polymyxin B. Of the $74 \mathrm{~A}$. baumannii isolates, 14 (18.92\%) were classified as MDR, 38 (51.35\%) were classified as XDR, and $22(29.73 \%)$ of the isolates were non-MDR. As such, $\sim 70.0 \%$ of the isolates were either MDR or XDR (Figure 4B). All clinical isolates grew well on CHROMagar Acinetobacter supplemented with MDR selective supplement, whereas all soil isolates were not culturable on this medium.

\section{Genotypic and phenotypic determination of MBL}

Although MBL enzymes were detected phenotypically in some clinical isolates, MBL genes $\left(b l a_{\mathrm{VIM}}, b l a_{\mathrm{IMP}}\right.$, and $\left.b l a_{\mathrm{SIM}}\right)$ were not detected neither in clinical nor in soil isolates (data not shown).

\section{Biofilm production assay}

In CRA method, out of eight positive isolates, 7 (9.46\%) displayed black colonies with dry crystalline morphology. The remaining 66 (89.19\%) isolates exhibited pink colonies 
Figure 1. Identification of $A$. baumannii isolates. (A) $A$. baumannii colonies on blood agar. (B) Non-lactose-fermenting colonies of A. baumannii on MacConkey agar. (C) A. baumannii metallic reddish colonies on CHROMagar Acinetobacter. (D) Non-lactose fermenter $A$. baumannii colonies appear lavender in color on Herellea agar. (E) Biochemical identification of $A$. baumannii by API-20E system with 7-digit number (0004042) according to analytical index
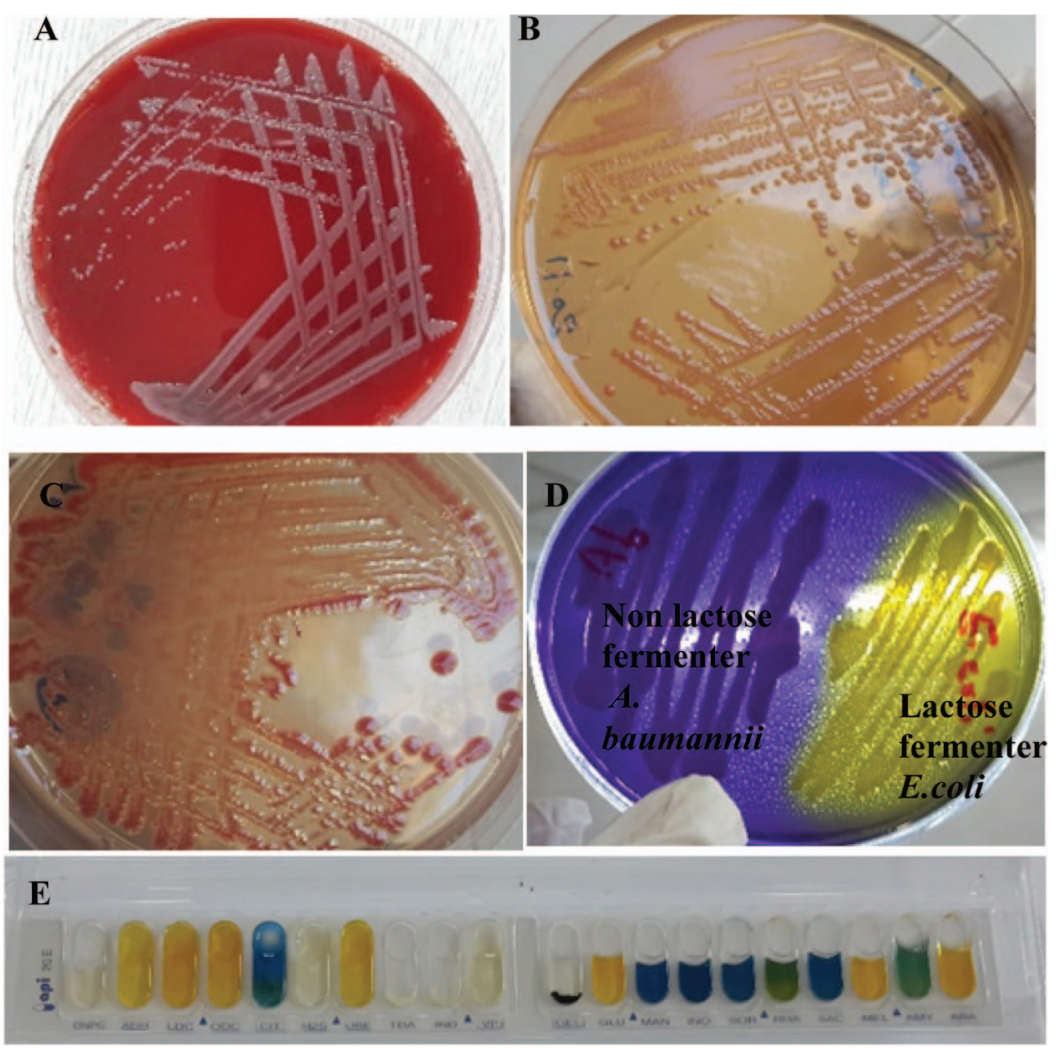

Figure 2. PCR products of the bla 0 XA-51-like gene in $A$. baumannii. Lane 1: 100-bp ladder; Lanes 2-6 were related to the clinical and soil isolates; Lane 7: positive control (A. baumannii ATCC 19606); and Lane 8: negative control (sterile distilled water)

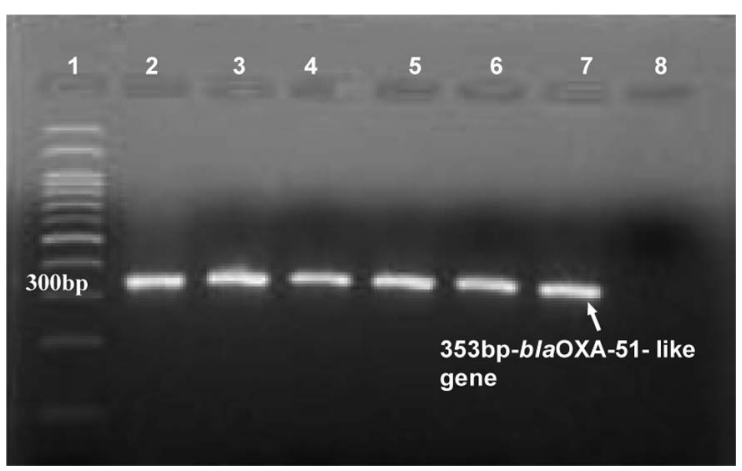

(as a non-biofilm producer), when cultured on CRA medium (Figure 5A), but most of these isolates produce biofilms in tube and TCP methods.

In the $\mathrm{TM}$, the rate of the non-biofilm producer was 27 (36.49\%) isolates and biofilm producers were 47 (63.51\%) (Table II and Figure 5B).

The results of biofilm formation in the TCP method showed that $64(86.49 \%)$ isolates could produce biofilms and only $10(13.51 \%)$ isolates were not able to produce
Figure 3. Clinical sources of $A$. baumannii isolates

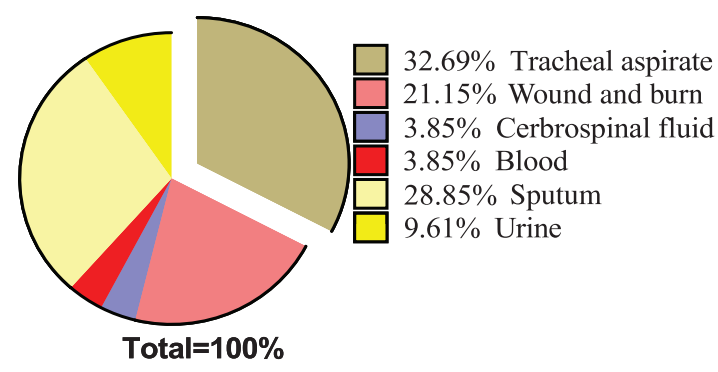

biofilms (Table II). The soil isolates produced higher rate (36.36\%) of strong biofilms than the clinical isolates (3.84\%) as showed in Table III.

The results of biofilm formation by the TCP method were used to study if there is any relation between biofilm production and drug resistance in clinical and soil isolates. The mean $\mathrm{OD}_{490}$ value for the reference strain (A. baumannii ATCC 19606) was $0.472 \pm 0.024$. The mean $\mathrm{OD}_{490}$ value for the negative control (wells inoculated with sterile broth were used as negative control) was $0.121 \pm 0.036$ and this value was used as the ODc. The classification of biofilm based on ODc revealed that $\mathrm{OD}_{490} \leq 0.121=$ non-biofilm; $0.121<$ $\mathrm{OD}_{490} \leq 0.242=$ weak biofilm, $0.242<\mathrm{OD}_{490} \leq 0.484=$ moderate biofilm; $\mathrm{OD}_{490}>0.484=$ strong biofilm. The median $\mathrm{OD}_{490}$ and IQR value for non-biofilm formers was $0.112(0.104,0.119)$, for weak biofilm formers was 0.183 
Table I. Antibiotic susceptibility pattern of clinical and soil A. baumannii isolates

\begin{tabular}{|c|c|c|c|c|c|c|}
\hline \multirow[b]{2}{*}{ Antibiotic disks } & \multicolumn{2}{|c|}{ Sensitive } & \multicolumn{2}{|c|}{ Intermediate } & \multicolumn{2}{|c|}{ Resistance } \\
\hline & Clin. no. (\%) & Soil no. (\%) & Clin. no. (\%) & Soil no. (\%) & Clin. no. (\%) & Soil no. (\%) \\
\hline AK & 4 (7.69) & $8(36.36)$ & $7(13.46)$ & $8(36.36)$ & 41 (78.85) & $6(27.28)$ \\
\hline MEM & $0(0)$ & $22(100)$ & $4(7.69)$ & $0(0)$ & $48(92.31)$ & $0(0)$ \\
\hline IMP & $0(0)$ & $22(100)$ & $2(3.85)$ & $0(0)$ & 50 (96.15) & $0(0)$ \\
\hline PB & $52(100)$ & $22(100)$ & $0(0)$ & $0(0)$ & $0(0)$ & $0(0)$ \\
\hline CAZ & $0(0)$ & $8(36.36)$ & $0(0)$ & $0(0)$ & $52(100)$ & $14(63.64)$ \\
\hline AMC & $0(0)$ & $0(0)$ & $0(0)$ & $0(0)$ & $52(100)$ & $22(100)$ \\
\hline TMP & $1(1.92)$ & $0(0)$ & $3(5.77)$ & $0(0)$ & $48(92.31)$ & $22(100)$ \\
\hline CIP & $0(0)$ & $20(90.1)$ & $0(0)$ & $0(0)$ & $52(100)$ & $2(9.09)$ \\
\hline PRL & $0(0)$ & $2(9.09)$ & $0(0)$ & $0(0)$ & $52(100)$ & $20(90.91)$ \\
\hline $\mathrm{CN}$ & $2(3.85)$ & $22(100)$ & $0(0)$ & $0(0)$ & $50(96.15)$ & $0(0)$ \\
\hline ATM & $0(0)$ & $0(0)$ & $3(5.77)$ & $0(0)$ & $49(94.23)$ & $22(100)$ \\
\hline
\end{tabular}

Note: AK: amikacin; MEM: meropenem; IMP: imipenem; PB: phosphate buffer; CAZ: ceftazidime; AMC: amoxicillin/clavulanic acid; TMP: trimethoprim; CIP: ciprofloxacin; PRL: piperacillin; CN: gentamicin; ATM: aztreonam.

Figure 4. Antibiotic-resistant phenotypes of $A$. baumannii isolates examined in this study. (A) The antibiotic susceptibility patterns of clinical (cli) and soil (so) A. baumannii isolates to tested antibiotics. (B) Approximately $70 \%$ of the isolates exhibited multidrug and extensively drug resistance, and approximately $30 \%$ revealed as non-MDR and all of them from soil isolates. S: sensitive; I: intermediate; R: resistance, Amo/ Clavu: amoxicillin/clavulanicacid

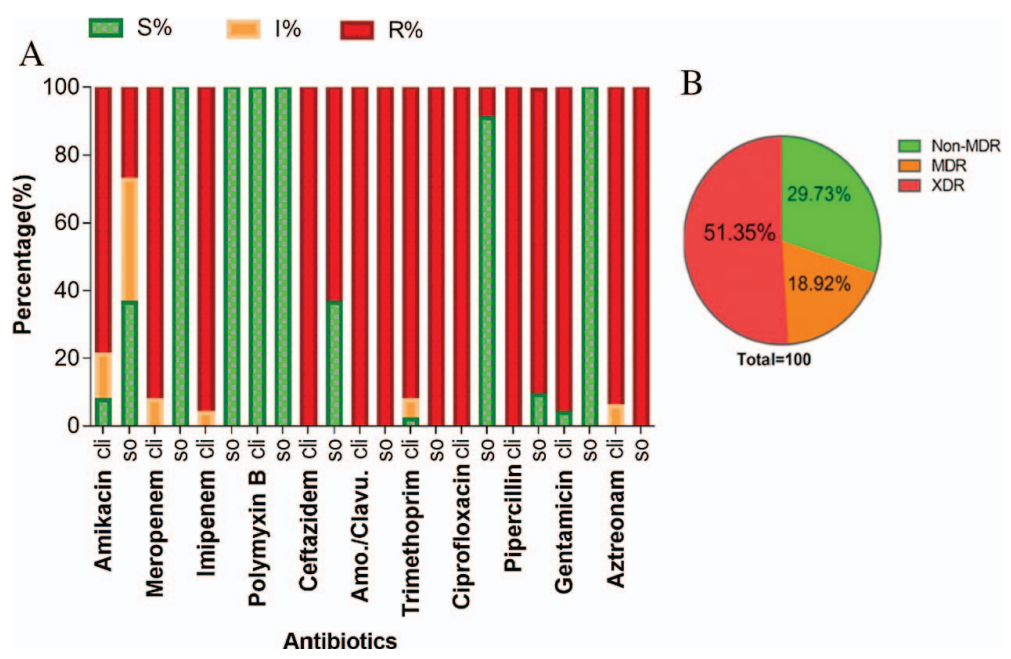

(0.142, 0.230), for moderate biofilm formers was 0.349 $(0.311,0.365)$, and for strong biofilm formers 0.498 $(0.490,0.572)$. All carbapenems (MEM and IMP) susceptible A. baumannii isolates were from soil isolates, and $36.36 \%$ of them tended to form stronger biofilms than resistant strains $(4.16 \%)$ of the clinical isolates (Figure 6).

To investigate whether there is any relationship between the formation of biofilm and antibiotic resistance, we first evaluated the composition of the biofilm-producing groups with resistance phenotypes. Among the 10 strong biofilm formers, $80 \%$ were non-MDR isolates and $20 \%$ were MDR/ XDR ones. The 16 weak biofilm formers consisted of $12.5 \%$ non-MDR, 56.25\% XDR, and 31.25\% MDR isolates. Ten strains that were negative for biofilm formation consisted of $20 \%$ non-MDR and $80 \%$ MDR/XDR isolates (Figure 7). As observed, the population that revealed more robust biofilm formation expected to contain larger proportion of non-MDR isolates.

The correlation between biofilm and resistance to the six antibiotics was analyzed. For four of them (MEM, IMP, 
Figure 5. Detection of biofilm producer A. baumannii isolates. (A) Growth on CRA medium. (A - 1): Black-colored colonies with dry crystalline consistency is indicative of strong biofilm forming phenotype. (B) Tube method: Tubes 1, 2, and 7 with moderate biofilm formation; Tube 3 for positive control ATCC A. baumannii strain; Tube 4 for negative control; and Tubes 5 and 6 strong biofilm former isolates. (C) The tissue culture plate method: 1 - negative control, 2 and 4 - strong biofilm former isolates, 3 and 7 - moderate, 5 for $A$. baumannii ATCC 19606 strain, and 6 weak biofilm producer isolate

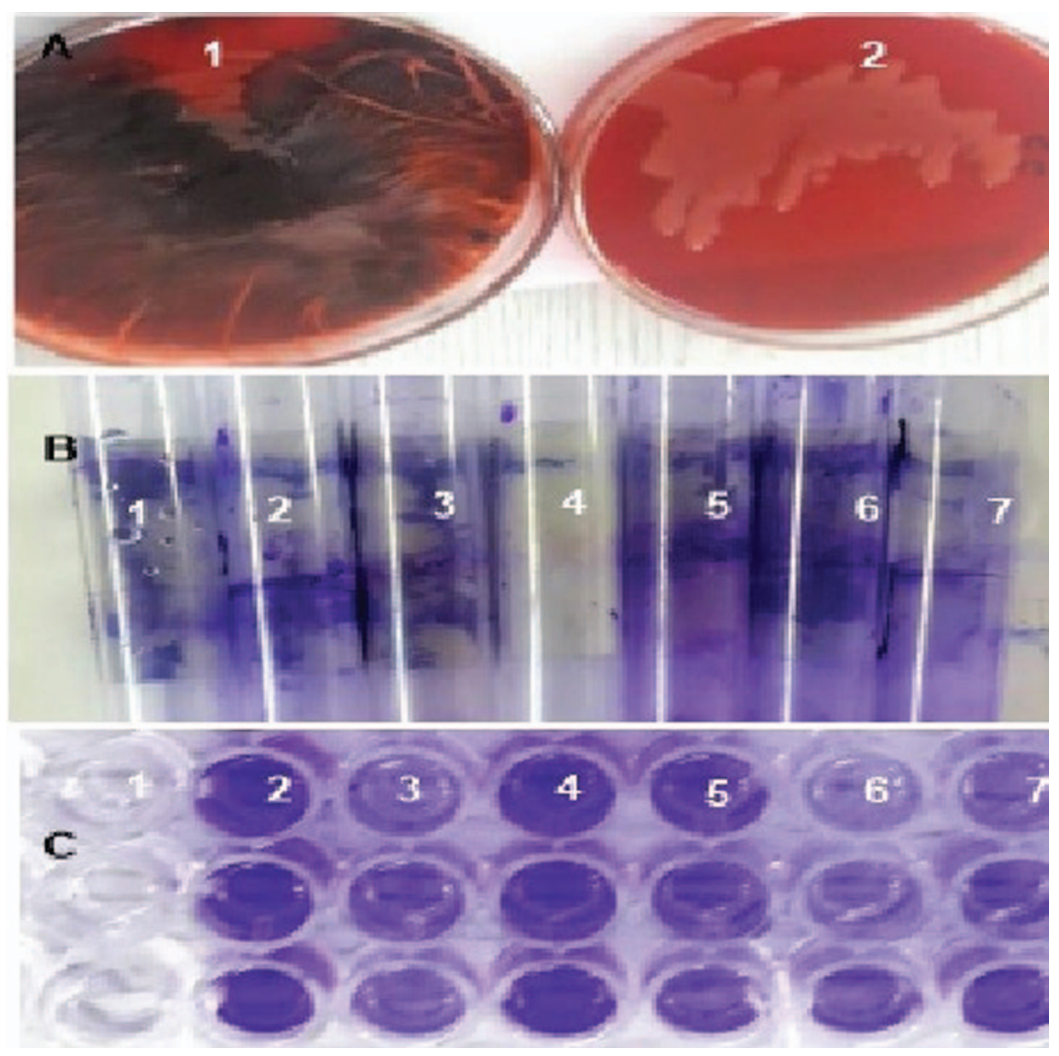

Table II. Screening of $A$. baumannii isolates for biofilm formation by tissue culture plate (TCP), tube method (TM), and Congo red agar (CRA)

\begin{tabular}{|l|c|c|c|c|}
\hline & Biofilm formation & TCPM [n (\%)] & TM [n (\%)] & CRA [n (\%)] \\
\hline \multirow{3}{*}{ No. of isolates (74) } & High & $10(13.51)$ & $9(12.16)$ & $7(9.46)$ \\
\cline { 2 - 5 } & Moderate & $38(51.35)$ & $28(37.84)$ & $1(1.35)$ \\
\cline { 2 - 5 } & Weak & $16(21.62)$ & $10(13.51)$ & $0(0)$ \\
\cline { 2 - 5 } & None & $10(13.51)$ & $27(36.49)$ & $66(89.19)$ \\
\hline
\end{tabular}

Table III. Characterization of biofilm status in clinical and soil isolates of $A$. baumannii

\begin{tabular}{|l|c|c|c|}
\hline Biofilm production & Clinical isolates (CRAB) [n (\%)] & Soil isolates [n (\%)] & Total no. (\%) \\
\hline Non & $8(15.38)$ & $2(9.09)$ & $10(13.51)$ \\
\hline Weak & $14(26.92)$ & $2(9.09)$ & $16(21.62)$ \\
\hline Moderate & $28(53.84)$ & $10(45.46)$ & $38(51.35)$ \\
\hline Strong & $2(3.84)$ & $8(36.36)$ & $10(13.51)$ \\
\hline Total & $52(100)$ & $22(100)$ & $74(100)$ \\
\hline
\end{tabular}

Note: CRAB: carbapenem-resistant Acinetobacter baumannii. 
Figure 6. Characterization of biofilm production in 48 clinical carbapenem-resistant (CR) and 22 soil carbapenem-sensitive (CS) isolates of $A$. baumannii (AB). Each individual bar represents the proportion that contain different biofilm status

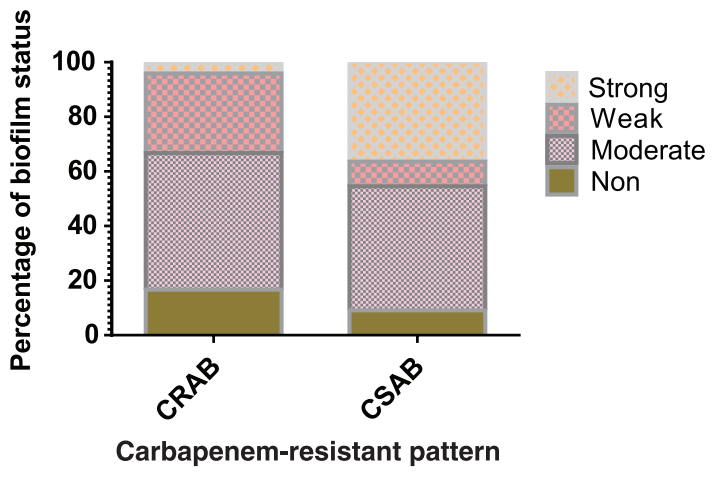

Figure 7. A percentage stacked bar graph displaying the distribution of non-susceptible phenotypes among different $A$. baumannii biofilm production capacities. Population that showed strong biofilm formation expected larger proportion of non-MDR isolates

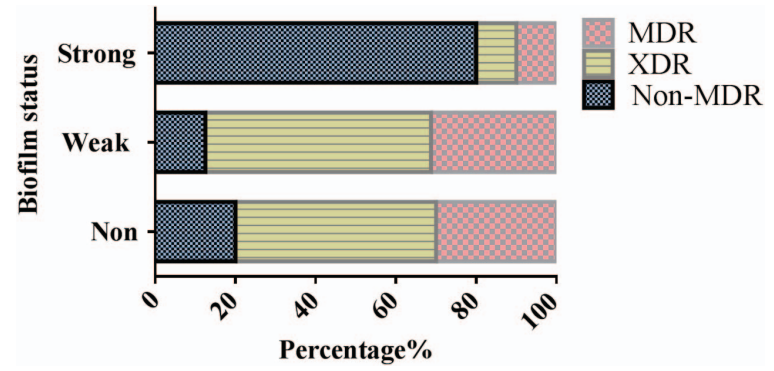

CIP, and GEN), susceptible isolates could form stronger biofilms than non-susceptible ones $(p<0.05$; MannWhitney $U$ test; Figure 8A-D). While for TMP and AK, no significant difference in biofilm formation between sensitive and resistance isolates was observed (Figure $8 \mathrm{E}$ and $\mathrm{F}$ ). We presume this was due to huge sample size difference, 70 isolates were non-susceptible to CAZ and only 4 were susceptible. For AK, 47 isolates were nonsusceptible and only 12 were susceptible. These results were also confirmed to compare the median (IQR) of $\mathrm{OD}_{490}$ between antimicrobial drug-resistant and susceptible groups (Table IV).

\section{DISCUSSION}

In the past few years, nosocomial infections caused by $A$. baumannii have become a major challenge for healthcare systems and treatments of infections caused by MDR strains are major concerns $[17,18]$. In this study 350 clinical samples were collected, and altogether $52(14.86 \%)$ isolates were $A$. baumannii that represent importance of this bacterium as a nosocomial pathogen in our hospitals.
These results were concurred with other studies conducted in Iraq $[19,20]$ and Iran [21]. The high prevalence of this bacteria observed in this study is probably related to nonobedience with the recommendations for control of the hospital environment [22], lack of hand hygiene, and misuse of antibiotics [23]. Some studies have shown that this microorganism, which has emerged worldwide as a pathogen causing severe infections in hospitalized patients, can persist in the environment for a long period of time, colonize patients or healthy individuals, and can at any time cause infection [24].

In the current research, $b l a_{\text {OXA-51-like }}$ gene was detected in all isolates, this gene is known as omnipresent and intrinsic to A. baumannii species [8, 25].

In this study, most isolates were recovered from tracheal aspirate $(32.69 \%)$ followed by sputum $(28.85 \%)$ and burned wound (21.15\%). Amudhan et al. [26] found that the highest percentage of isolation was from respiratory secretions followed by blood and wounds. Furthermore, Martins et al. [27] found that the majority of isolates $(66.4 \%)$ was obtained from the respiratory tract, followed by blood (9.67\%) and urine $(8.4 \%)$ cultures.

In this study, antibiotic resistance rates of A. baumannii clinical isolates from hospitalized patients were very high, especially against AMC, PRL, CIP, CAZ, IMP, GEN, ATM, and MEM. These results disagree with results obtained by Jabour [28], who has reported a low resistant rate of $A$. baumannii isolates to PRL (20\%), AK and GEN (40\%), CIP $(30 \%)$ and IMP $(30 \%)$. Furthermore, our results are relatively in accordance with Ayan et al. [29], who reported that from 52 clinical isolates, all strains were resistant to PRL, CAZ, GEN, and ATM. The results of this study also showed high resistant rate of clinical isolates to IMP and MEM, similar findings exhibited by a study conducted on Arab League countries [30], which showed that the highest percentage of carbapenem-resistant A. baumannii was recorded in Iraq (89\%).

Analysis of antibiotic resistance patterns showed that all the 52 clinical isolates of A. baumannii were MDR and XDR. Such resistance ascribed to the extensive use of broadspectrum antibiotics in clinical settings $[31,32]$.

Our results indicated that soil A. baumannii isolates exhibited $100 \%$ resistance to AMC, TMP, and ATM and 90.91\% resistance to PRLPRL. However, AMC, ATM, and PRL are $\beta$-lactam antibiotics and all soil strains in this study showed negative for $b l a_{\mathrm{OXA}-23}$ and MBL enzymes $\left(b l a_{\mathrm{VIM}}\right.$, $b l a_{\mathrm{IMP}}$, and $\left.b l a_{\mathrm{SIM}}\right)$. The increased resistance of soil strains to AMC, ATM, and PRL was not intriguing, as these antibiotics belong to the family of $\beta$-lactams, and most of them originate from natural drugs that are present in the environment. For this reason, several reports confirm that antibiotics are produced at adequately high concentrations in soil to inhibit bacterial growth in the area of the producers [33-35]. These results suggest that soil habitants should be expected to impose a selective pressure to drive the development of antibiotic resistance mechanisms as an essential survival strategy to defend against antibioticproducing strains. 
Figure 8. Relationship between biofilm formation and the resistance of clinical and soil $A$. baumannii isolates to six antibiotics. Optical density at $490 \mathrm{~nm}$ represents biofilm-forming capacity. (A-D) For meropenem (MEM), imipenem (IMP), ciprofloxacin (CIP), and gentamicin (GEN), susceptible isolates tended to form stronger biofilms (higher OD values) than non-susceptible ones. (E-F) For trimethoprim (TMP) and amikacin (AK), no significant difference in biofilm production among susceptible and resistant isolates was observed (Mann-Whitney $U$ test)
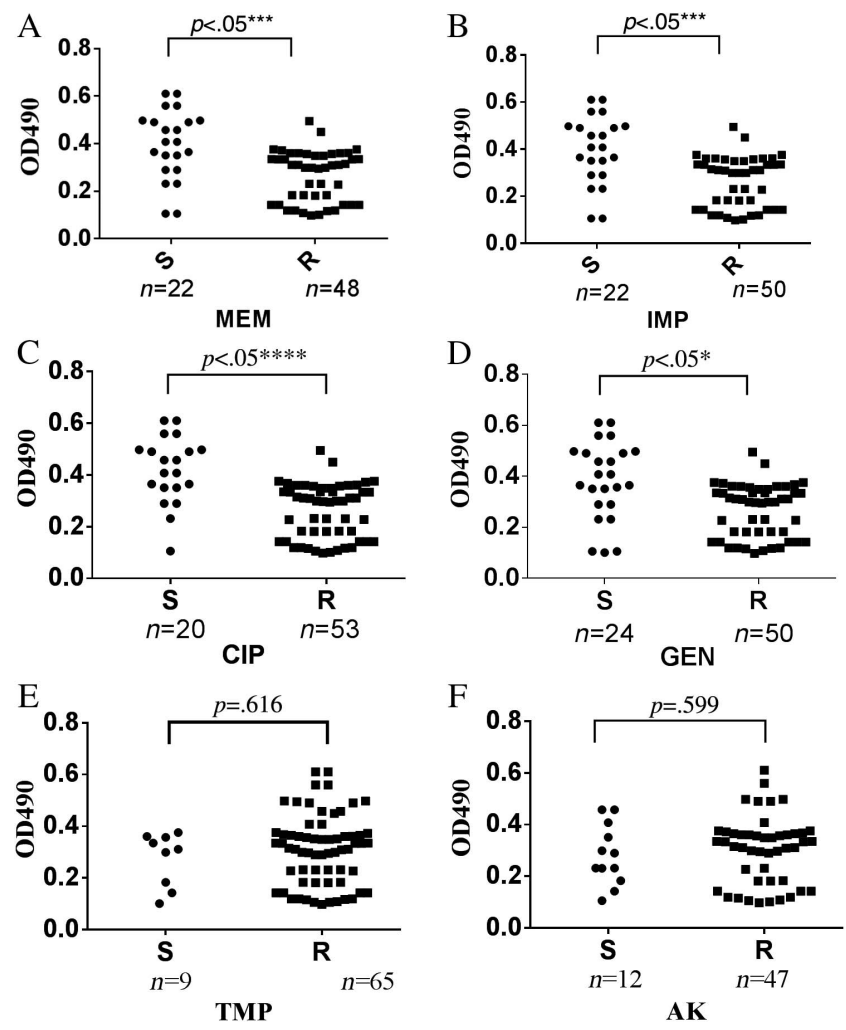

Table IV. Correlation between the biofilm value $\left(0 \mathrm{D}_{490}\right)$ and drug-resistance phenotype in all (clinical and soil) $A$. baumannii strains

\begin{tabular}{|c|c|c|c|c|}
\hline \multirow[b]{2}{*}{ Antimicrobial group } & \multirow[b]{2}{*}{ Antimicrobial } & \multicolumn{2}{|c|}{ Median (IQR) $0 D_{490}$} & \multirow[b]{2}{*}{$p$ value } \\
\hline & & Susceptible & Resistant & \\
\hline \multirow[t]{2}{*}{ Carbapenems } & Imipenem & $0.408(0.290,0.498)$ & $0.299(0.152,0.349)$ & $<0.05^{*}$ \\
\hline & Meropenem & $0.408(0.290,0.498)$ & $0.299(0.152,0.349)$ & $<0.05^{*}$ \\
\hline \multirow[t]{2}{*}{ Aminoglycosides } & Gentamicin & $0.386(0.290,0496)$ & $0.299(0.172,0.349)$ & $<0.05^{*}$ \\
\hline & Amikacin & $0.261(0.195,0.393)$ & $0.334(0.182,0.365)$ & $0.54^{*}$ \\
\hline Fluoroquinolones & Ciprofloxacin & $0.408(0.320,0.498)$ & $0.299(0.162,0.349)$ & $<0.05^{*}$ \\
\hline Cephems & Ceftazidime & $0.433(0.375,0.482)$ & $0.311(0.182,0.360)$ & $<0.05^{*}$ \\
\hline Penicillins & Piperacillin & - & $0.313(0.183,0.365)$ & - \\
\hline $\begin{array}{l}\text { Penicillins }+\beta \text {-lactamase } \\
\text { inhibitors }\end{array}$ & Amoxicillin/clavulanic acid & - & $0.313(0.183,0.365)$ & - \\
\hline Folate pathway inhibitor & Trimethoprim & $0.311(0.162,0.358)$ & $0.315(0.183,0.370)$ & 0.6165 \\
\hline Monobactam & Aztreonam & - & $0.313(0.183,0.365)$ & - \\
\hline Lipopeptide & Polymyxin B & $0.313(0.183,0.365)$ & - & - \\
\hline
\end{tabular}

Note: Optical density at $490 \mathrm{~nm}\left(\mathrm{OD}_{490}\right)$ data shown in median [interquartile range (IQR)]. The values of $p$ represent the comparison of median $0 \mathrm{D}_{490}$ of bacterial isolates between two groups (Mann-Whitney $U$ test). An asterisk $\left(^{*}\right)$ indicates the significance $(p$ value). 
Of the 11 antimicrobials tested, the most powerful one was polymyxin B (100\%) for all clinical and environmental isolates. In agreement with Mak et al. [31] who requested, the most effective drug in controlling A. baumannii is polymyxin B.

Only 8 out of the total 74 isolates that were cultured on the CRA medium produced black colony. However, in the TM, 47 (63.51\%) isolates were biofilm producers. The results of biofilm formation in the TCP method showed that $86.49 \%$ of the isolates were biofilm producer. In accordance with the previous studies, CRA method cannot be suggested as a general screening test to detect biofilm-producing isolates $[36,37]$.

The TM harmonized well with TCP for identifying strong biofilm producers, but it was difficult to differentiate between moderate and weak because of the variability in the results detected by different observers. Hence, based on the previous reports, TCP is a quantitative, reliable and gold standard method to identify biofilm-producing isolate [14, 37].

The prevalence of biofilm-producing strains among the soil A. baumannii isolates were higher than $90 \%$, in which $100 \%$ of non-MDR and $80 \%$ of all strong biofilm former were non-MDR strains. As observed, more non-resistant isolates intrinsically tended to produce stronger biofilms. Qi et al. [38] reported in their study that biofilm acts as a mechanism for bacteria to obtain a better survival, particularly in cases when resistance level is low. The relationship between the biofilm value and individual drug resistance of all A. baumannii isolates (clinical and soil) indicates that the isolates susceptible to MEM, IMP, CIP, CAZ, and GEN tend to form stronger biofilms (higher $\mathrm{OD}_{490}$ value) than the resistant strains $(p<0.05$; Mann-Whitney $U$ test). Rodriguez-Bano et al. [39] reported that A. baumannii biofilm-forming isolates were (less resistant to IMP and CIP) unlike non-biofilm-forming strains; these strains are not dependent on antimicrobial resistance for survival, which is comparable with our results.

However, in contrast to our results, Thummeepak et al. [40] concluded that high-level GEN-resistant strains show larger biofilm biomass compared with those strains, which have low-level resistance.

\section{CONCLUSIONS}

The prevalence of antibiotic resistance pattern in A. baumannii of soil isolates is less than clinical isolates. These differences may be due to excessive use of drugs in human beings, an uncompleted period of antibiotic therapy and incorrect use of detergents and disinfectants that all these lead to increased mutagenesis in bacteria recently. In addition, this study showed that most clinical and soil isolates of A. baumannii have the ability to produce biofilms. However, the soil isolates produce more robust biofilms (help them to persist in new environments) than clinical isolates and consequently could lead to a change of soil isolates from non-MDR to MDR when transmitted to the hospital environment. Finally, the non-MDR strains tend to form stronger biofilms than the MDR and XDR strains.
Conflict of Interest: The authors declare no conflict of interest.

\section{REFERENCES}

1. Bergogne-Berezin, E., Towner, K. J.: Acinetobacter spp. as nosocomial pathogens: Microbiological, clinical, and epidemiological features. Clin Microbiol Rev 9, 148-165 (1996).

2. Zarrilli, R., Crispino, M., Bagattini, M., Barretta, E., Di Popolo, A., Triassi, M., Villari, P.: Molecular epidemiology of sequential outbreaks of Acinetobacter baumannii in an intensive care unit shows the emergence of carbapenem resistance. J Clin Microbiol 42, 946-953 (2004).

3. Harding, C. M., Hennon, S. W., Feldman, M. F.: Uncovering the mechanisms of Acinetobacter baumannii virulence. Nat Rev Microbiol 16, 91-102 (2018).

4. Hall-Stoodley, L., Costerton, J. W., Stoodley, P.: Bacterial biofilms: From the natural environment to infectious diseases. Nat Rev Microbiol 2, 95-108 (2004).

5. Singhai, M., Malik, A., Shahid, M., Malik, M. A., Goyal, R.: A study on device-related infections with special reference to biofilm production and antibiotic resistance. J Glob Infect Dis 4, 193-198 (2012).

6. Dunne, W. M.: Bacterial adhesion: Seen any good biofilms lately? Clin Microbiol Rev 15, 155-166 (2002).

7. Hoiby, N., Ciofu, O., Johansen, H. K., Song, Z. J., Moser, C., Jensen, P. O., Molin, S., Givskov, M., Tolker-Nielsen, T., Bjarnsholt, T.: The clinical impact of bacterial biofilms. Int J Oral Sci 3, 55-65 (2011).

8. Turton, J. F., Woodford, N., Glover, J., Yarde, S., Kaufmann, M. E., Pitt, T. L.: Identification of Acinetobacter baumannii by detection of the $b l a_{\mathrm{OXA}-51-\text { like }}$ carbapenemase gene intrinsic to this species. J Clin Microbiol 44, 2974-2976 (2006).

9. Woodford, N., Ellington, M. J., Coelho, J. M., Turton, J. F., Ward, M. E., Brown, S., Amyes, S. G., Livermore, D. M.: Multiplex PCR for genes encoding prevalent OXA carbapenemases in Acinetobacter spp. Int J Antimicrob Agents 27, 351-353 (2006).

10. Clinical and Laboratory Standards Institute: Performance Standards for Antimicrobial Susceptibility Testing, $27^{\text {th }}$ Edition. CLSI Supplement M100. Clinical and Laboratory Standards Institute, Wayne, PA, 2017.

11. Manchanda, V., Sanchaita, S., Singh, N. P.: Multidrug resistant Acinetobacter. J Global Infect Dis 2, 291-304 (2010).

12. Christensen, G. D., Simpson, W. A., Bisno, A. L., Beachey, E. H.: Adherence of slime-producing strains of Staphylococcus epidermidis to smooth surfaces. Infect Immun 37, 318-326 (1982).

13. Christensen, G. D., Simpson, W. A., Younger, J. A., Baddor, M. A., Barrett, F. F., Melton, M. D., Beachy, H. E.: Adherence of coagulase negative Staphylococci to plastic tissue cultures: A quantitative model for the adherence of Staphylococci to medical devices. J Clin Microbiol 22, 996-1006 (1985).

14. Mathur, T., Singhal, S., Khan, S., Upadhyay, D. J., Fatma, T., Rattan, A.: Detection of biofilm formation among the clinical isolates of Staphylococci: An evaluation of three different screening methods. Indian J Med Microbiol 24, 25-29 (2006). 
15. Zhang, D., Xia, J., Xu, Y., Gong, M., Zhou, Y., Xie, L., Fang, X.: Biological features of biofilm-forming ability of Acinetobacter baumannii strains derived from 121 elderly patients with hospital-acquired pneumonia. Clin Exp Med 16, 73-80 (2016).

16. Freeman, D. J., Keane, C. T.: New method for detecting slime production by coagulase negative staphylococci. J Clin Pathol 42, 872-874 (1989).

17. Tang, S. S., Apisarnthanarak, A., Hsu, L. Y.: Mechanisms of beta-lactam antimicrobial resistance and epidemiology of major community- and healthcare-associated multidrug-resistant bacteria. Adv Drug Deliv Rev 78, 3-13 (2014).

18. Peleg, A. Y., Seifert, H., Paterson, D. L.: Acinetobacter baumannii: Emergence of a successful pathogen. Clin Microbiol Rev 21, 538-582 (2008).

19. Al Sehlawi, A. M., Al Thahab, A. A.: Isolation and identification of Acinetobacter baumannii clinical isolates using novel methods. J Pure Appli Sci 22, 1041-1050 (2014).

20. Al-Kadmy, I. M. S., Ali, A. N. M., Salman, I. M. A., Khazaal, S. S.: Molecular characterization of Acinetobacter baumannii isolated from Iraqi hospital environment. New Microbes New Infect 21, 51-57 (2018).

21. Babapour, E., Haddadi, A., Mirnejad, R., Angaji, S. A., Amirmozafari, N.: Biofilm formation in clinical isolates of nosocomial Acinetobacter baumannii and its relationship with multidrug resistance. Asian Pac J Trop Biomed 6, 528-533 (2016).

22. Huang, C. H., Lee, C. L., Lin, A. C. M., Chen, W. Y., Teng, P. C., Lee, S. H., Hsieh, Y. J., Jang, T. N.: Different strains of Acinetobacter baumannii spreading in an intensive care unit. J Acute Med 1, 5-10 (2011).

23. Fishbain, J., Peleg, A. Y.: Treatment of Acinetobacter infections. Clin Infect Dis 51, 79-84 (2010).

24. Jung, J. Y., Park, M. S., Kim, S. E., Park, B. H., Son, J. Y., Kim, E. Y., Lim, J. E., Lee, S. K., Lee, S. H., Lee, K. J., Kang, Y. A., Kim, S. K., Chang, J., Kim, Y. S.: Risk factors for multi-drug resistant Acinetobacter baumannii bacteremia in patients with colonization in the intensive care unit. BMC Infect Dis 10, 1-11 (2010).

25. Khorsi, K., Messai, Y., Hamidi, M., Ammari, H., Bakour, R.: High prevalence of multidrug-resistance in Acinetobacter baumannii and dissemination of carbapenemase-encoding genes $b l a_{\mathrm{OXA}-23-\mathrm{like}}, b l a_{\mathrm{OXA}-24-\text { like }}$ and $b l a_{\mathrm{NDM}-1}$ in Algiers hospitals. Asian Pac J Trop Med 8, 438-446 (2015).

26. Amudhan, S. M., Sekar, U., Arunagiri, K., Sekar, B.: OXA beta-lactamase-mediated carbapenem resistance in Acinetobacter baumannii. Indian J Med Microbiol 29, 269-274 (2011).

27. Martins, A. F., Kuchenbecker, R. S., Pilger, K. O., Pagano, M., Barth, A. L.: CMCIES-PMPA/SMS Task Force: High endemic levels of multidrug-resistant Acinetobacter baumannii among hospitals in southern Brazil. Am J Infect Control 40, 108-112 (2012).

28. Jabur, M. H.: Isolation of Acinetobacter baumannii from different clinical source and study some antibiotic resistant and $\beta$-lactamase production. Med J Babylon 11, 456-464 (2014).

29. Ayan, M., Durmaz, R., Aktas, E., Durmaz, B.: Bacteriological, clinical and epidemiological characteristics of hospitalacquired Acinetobacter baumannii infection in a teaching hospital. J Hosp Infect 54, 39-45 (2003).

30. Moghnieh, R. A., Kanafani, Z. A., Tabaja, H. Z., Sharara, S. L., Awad, L. S., Kanj, S. S.: Epidemiology of common resistant bacterial pathogens in the countries of the Arab League. Lancet Infect Dis 18, e379-e394 (2018).

31. Mak, J. K., Kim, M. J., Pham, J., Tapsall, J., White, P. A.: Antibiotic resistance determinants in nosocomial strains of multidrug-resistant Acinetobacter baumannii. J Antimicrob Chemother 63, 47-54 (2009).

32. Liu, S., Wang, Y., Xu, J., Li, Y., Guo, J., Ke, Y., Yuan, X., Wang, L., Du, X., Wang, Z., Huang, L., Zhang, N., Chen, Z.: Genome sequence of an OXA23-producing, carbapenem-resistant Acinetobacter baumannii strain of sequence type ST75. J Bacteriol 194, 6000-6001 (2012).

33. Anukool, U., Gaze, W. H., Wellington, E. M.: In situ monitoring of streptothricin production by Streptomyces rochei F20 in soil and rhizosphere. Appl Environ Microbiol 70, 5222-5228 (2004).

34. Hansen, L. H., Ferrari, B., Sorensen, A. H., Veal, D., Sorensen, S. J.: Detection of oxytetracycline production by Streptomyces rimosus in soil microcosms by combining whole-cell biosensors and flow cytometry. Appl Environ Microbiol 67, 239-244 (2001).

35. Li, D. M., Alexander, M.: Factors affecting co-inoculation with antibiotic-producing bacteria to enhance rhizobial colonization and nodulation. Plant Soil 129, 195-201 (1990).

36. Ruzicka, F., Hola, V., Votava, M., Tejkalova, R., Horvat, R., Heroldova, M., Woznicova, V.: Biofilm detection and the clinical significance of Staphylococcus epidermidis isolates. Folia microbiol 49, 596-600 (2004).

37. Hassan, A. U., Kaleem, F., Omair, M., Khalid, A., Iqbal, M.: Evaluation of different detection methods of biofilm formation in the clinical isolates. Braz J Infect Dis 15, 305-311 (2011).

38. Qi, L., Li, H., Zhang, C., Liang, B., Li, J., Wang, L., Du, X., Liu, X., Qiu, S., Song, H.: Relationship between antibiotic resistance, biofilm formation, and biofilm-specific resistance in Acinetobacter baumannii. Front Microbiol 7, 483 (2016).

39. Rodriguez-Bano, J., Marti, S., Soto, S., Fernandez-Cuenca, F., Cisneros, J. M., Pachon, J., Pascual, A., Martinez-Martinez, L., McQueary, C., Actis, L. A., Vila, J., Spanish Group for the Study of Nosocomial Infections: Biofilm formation in Acinetobacter baumannii: Associated features and clinical implications. Clin Microbiol Infect 14, 276-278 (2008).

40. Thummeepak, R., Kongthai, P., Leungtongkam, U., Sitthisak, S.: Distribution of virulence genes involved in biofilm formation in multi-drug resistant Acinetobacter baumannii clinical isolates. Int Microbiol 19, 121-129 (2016). 